\title{
Controlling the intensity of light in large areas at the interfaces of a scattering medium
}

\author{
Oluwafemi S. Ojambati, ${ }^{1, *}$ John T. Hosmer-Quint, ${ }^{1,2}$ Klaas-Jan Gorter, ${ }^{1}$ Allard P. Mosk,,${ }^{1,}$ and Willem L. Vos ${ }^{1}$ \\ ${ }^{1}$ Complex Photonic Systems (COPS), MESA+ Institute for Nanotechnology, University of Twente, \\ P.O. Box 217, 7500 AE Enschede, The Netherlands \\ ${ }^{2}$ Department of Physics, Lawrence University, 711 E. Boldt Way, Appleton, WI 54911, USA
}

(Received 12 July 2016; published 19 October 2016)

\begin{abstract}
The recent advent of wave-shaping methods has demonstrated the focusing of light through and inside even the most strongly scattering materials. Typically in wavefront shaping, light is focused in an area with the size of one speckle spot. It has been shown that the intensity is not only increased in the target speckle spot, but also in an area outside the optimized speckle spot. Consequently, the total transmission is enhanced, even though only the intensity in a single speckle spot is controlled. Here, we experimentally study how the intensity enhancement on both interfaces of a scattering medium depends on the optimization area on the transmission side. We observe that as the optimization radius increases, the enhancement of the total transmitted intensity increases. We find a concomitant decrease of the total reflected intensity, which implies an energy redistribution between transmission and reflection channels. In addition, we find qualitative evidence of a long-range reflectiontransmission correlation. Our result is useful for efficient light harvesting in solar cells, multichannel quantum secure communications, imaging, and complex beam delivery through a scattering medium.
\end{abstract}

DOI: 10.1103/PhysRevA.94.043834

\section{INTRODUCTION}

Wave interference in disordered scattering media results in speckles through the coherent addition of multiple waves, which are independent and have random amplitudes and phases [1]. Between these interfering waves, there exist short-, long-, and even infinite-range correlations [2-10]. These correlations have provided enriching information about mesoscopic transport, as well as a deeper understanding of fundamental phenomena such as enhanced backscattering [11-13] and Anderson localization [14,15].

In 1990, using speckle correlations, Freund predicted that an opaque scattering medium can be used as a lens and other optical elements by designing an appropriate incident wavefront [16]. Only recently, this prediction was confirmed by the advent of innovative wave-shaping methods such as wavefront shaping [17-22], time reversal [23-25], phase conjugation [26-28], and transmission-matrix-based control [29-31]. In wavefront shaping, an optimization algorithm receives as a feedback the intensity in a target area, typically one speckle spot with an area $A=\lambda^{2} / 2 \pi$ on the sample surface. The algorithm then modifies the spatial phase of the incident field on the scattering medium, such that the intensity in the target spot is maximized. These wave-shaping methods have led the way for exciting applications such as noninvasive biomedical imaging [32-34], advanced optics [35-40], and cryptography and secure communication $[41,42]$.

\footnotetext{
*o.s.ojambati@utwente.nl

${ }^{\dagger}$ Current address: Physics of Light in Complex Systems, Debye Institute for Nanomaterials Science, Utrecht University.
}

Published by the American Physical Society under the terms of the Creative Commons Attribution 3.0 License. Further distribution of this work must maintain attribution to the author(s) and the published article's title, journal citation, and DOI.
In an earlier experiment [18], an intensity enhancement was observed not only in the target speckle spot but also in an area outside the target speckle spot. Consequently, the total transmission was enhanced, even though only the intensity in a single speckle spot was monitored. An intuitive explanation for this observation is that there is a redistribution of energy between reflection and transmission channels, since absorption is negligible in the scattering samples. This observation was confirmed in Ref. [19]. Here, we take a further step by investigating how the enhancement of the total transmission depends on the optimization area. In the absence of absorption, we expect to observe a concomitant effect in the total reflected intensity. Moreover, we expect to find the effect of long-range correlations, especially of the form that exists between the reflected and transmitted speckles, as recently predicted in Ref. [43]. An optimization of the total intensity transmitted through a scattering medium, which is the extreme case of our study, has been performed in Refs. [19,44], although the optimization area was not systematically varied. The dependency that we seek will give insight into the intensity redistribution between the transmitted and reflected speckles. Such a fundamental understanding is useful for applications of wavefront shaping in efficient energy harvesting in solar cells [45-47], multichannel quantum secure communications [48,49], imaging [28,34,50,51], and the delivery of complex beams through a scattering medium [52].

In this paper, we experimentally study how the optimized intensity on both interfaces of a scattering medium depends on the optimization area on the transmission side. We image the transmitted intensity onto the chip of a camera, and thus there is a freedom to control the optimization radius. We observe that as the optimization radius increases, the enhancement of the total transmitted intensity increases. We find a concomitant decrease of the total reflected intensity, which implies that there is a redistribution of intensity from reflection to transmission. In addition, our result reveals qualitative evidence of the longrange reflection-transmission correlation. 


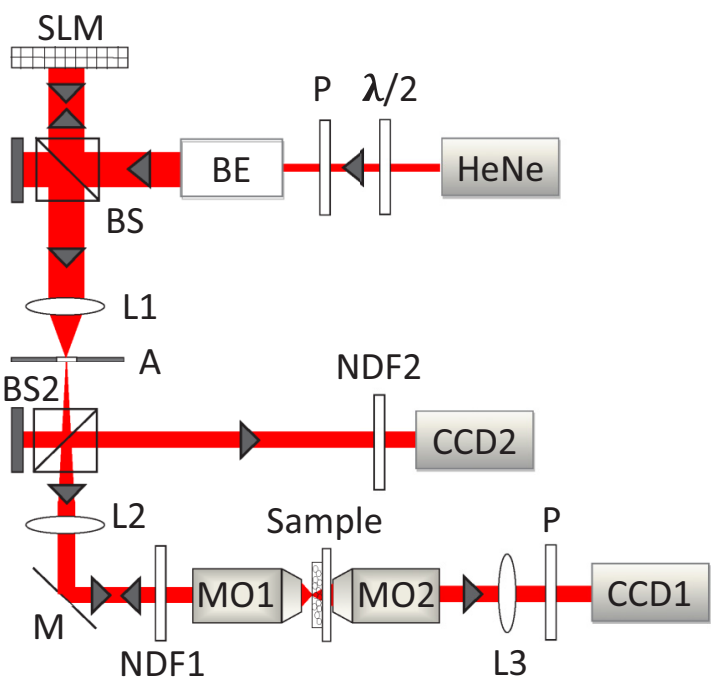

FIG. 1. Experimental setup. A HeNe laser beam is expanded and modulated by a spatial light modulator (SLM). The light is directed toward the microscope objective (MO1) (numerical aperture NA = 0.95 ) and then focused onto a multiple scattering sample. The sample is made of disordered $\mathrm{ZnO}$ nanoparticles. The intensity transmitted through the sample is imaged onto the chip of a charged-coupled device (CCD) camera (CCD1) through an oil-immersion objective $(\mathrm{NA}=1.42)$ and lens L3. The reflected intensity is also imaged through a focusing objective and lens L2 and detected by a CCD camera (CCD2). P: polarizer; $\lambda / 2$ : half-wave plate; $\mathrm{BE}$ : beam expander; BS: beam splitter; A: aperture; M: mirror; NDF: neutral density filter.

\section{EXPERIMENTAL DETAILS}

\section{A. Experiment setup}

The experimental setup is shown in Fig. 1. The light source is a helium neon $(\mathrm{HeNe})$ laser, which emits at a wavelength $\lambda=$ $632.8 \mathrm{~nm}$ and has an output power of $5 \mathrm{~mW}$. A combination of a half-wave plate $(\lambda / 2)$ and a polarizer $(\mathrm{P})$ controls the incident power and also fixes the polarization of the beam. A beam expander with a magnification of $20 \times$ expands the beam to fill the active area of the spatial light modulator (SLM). The SLM is a Holoeye Pluto (6010-NIR-011), which has $1920 \times 1080$ pixels and controls the horizontal polarization. A beam splitter (BS) separates the incident and reflected light from the SLM. The reflected light is focused by a lens L1 (focal length $f=250 \mathrm{~mm}$ ). Aperture A, placed at the focal plane of the lens L1, filters out the higher diffraction orders of the SLM's pixels and transmits only the 0 th order. With a telescope consisting of lenses L1 and L2 ( $f=250 \mathrm{~mm})$, the SLM is imaged onto the pupil of a microscope objective MO1 (Zeiss: infinity corrected, $63 \times, \mathrm{NA}=0.95)$, which focuses the light onto the surface of the sample. For a planar incident wavefront, the spot size is $0.35 \mu \mathrm{m}$. The sample is an ensemble of disordered zinc oxide nanoparticles that are spray painted on top of a glass cover slide. The sample thickness is $17 \pm 0.2 \mu \mathrm{m}$ and the transport mean free path $\ell$ of similar samples was reported in Ref. [53] to be $\ell=0.6 \pm 0.2 \mu \mathrm{m}$. The intensity transmitted through the sample is imaged onto the chip of a charged-coupled device (CCD) camera (CCD1) using a combination of an oil-immersion objective MO2 (Olympus: infinity corrected,
$60 \times, \mathrm{NA}=1.42)$ and lens $\mathrm{L} 3(f=500 \mathrm{~mm})$. The calculated magnification of imaging the back surface of the sample (M1) is $167 \times$. Similarly, a combination of the focusing objective MO1 and lens L2 images the reflected intensity exiting the front surface of the sample onto the chip of a CCD camera (CCD2). The calculated magnification on the reflection side is $95 \times$. Cameras CCD1 and CCD2 are both of the same type (AVT Dolphin 145B), with a pixel pitch of $6.45 \mu \mathrm{m}$. Using the calculated magnifications, the pixel pitches on the front and back surfaces of the sample are 0.068 and $0.038 \mu \mathrm{m}$ respectively. The neutral density filter NDF1 (Thorlabs NE05A), with a measured attenuation factor AF = 0.33 , attenuates the incident intensity on the sample in order to prevent saturation of the cameras. The reflected intensity is further attenuated by placing NDF2 (Thorlabs NE10A), with a measured $\mathrm{AF}=0.10$, in the reflection path. The reflected intensity is in total attenuated by a factor of 0.033 .

In the setup of Ref. [19], the optimization area was not controlled since the scattering sample was directly attached to a photodetector. Moreover, as a result of the refractive index contrast (approximately a factor of 2), the detected signal in Ref. [19] was limited by significant surface reflections between the scattering sample and photodector. With our setup, we have the freedom to control the optimization area. A further advantage of our study is that there is no significant surface reflection since there is an index match between the sample substrate and the immersion oil.

\section{B. Experimental procedure and parameters}

To optimize multiple speckle spots, we used the partitioning algorithm that is described in Ref. [54] rather than the stepwise sequential algorithm typically used in previous wavefront-shaping experiments [17-19,55,56]. We find that the partitioning algorithm outperforms the stepwise sequential and genetic algorithms for optimizing intensity in large areas (see Appendix A). In the optimization, the number of segments used is 5000. We systematically increased the number of transmission channels by increasing the optimization radius $r_{o}$. The number of open transmission channels $M$ scales linearly with the probed area $A\left(=\pi r_{o}^{2}\right)$

$$
M=\frac{2 \pi A n_{e}^{2}}{\lambda^{2}} \frac{\ell}{L},
$$

where $n_{e}$ is the effective refractive index of the scattering medium [57,58]. For a specific optimization radius, we repeated the wavefront-shaping experiment for two to five times at a fixed position on the sample. (Since the optimization time is longer for larger radii, we took fewer measurements). As a reference phase pattern, we sent 100 randomly generated patterns with the same number of segments as the optimized pattern. Compared to the optimized pattern, these randomly generated phase patterns had diffraction losses and a power incident on the sample that was larger by only $5 \%$, which underestimated the intensity enhancement by this amount.

To quantify the enhancement $\eta_{\text {targ }}$ of the total intensity in the target area, we define

$$
\eta_{\operatorname{targ}} \equiv \frac{P_{\operatorname{targ}}^{o}}{\left\langle P_{\operatorname{targ}}^{u}\right\rangle}
$$



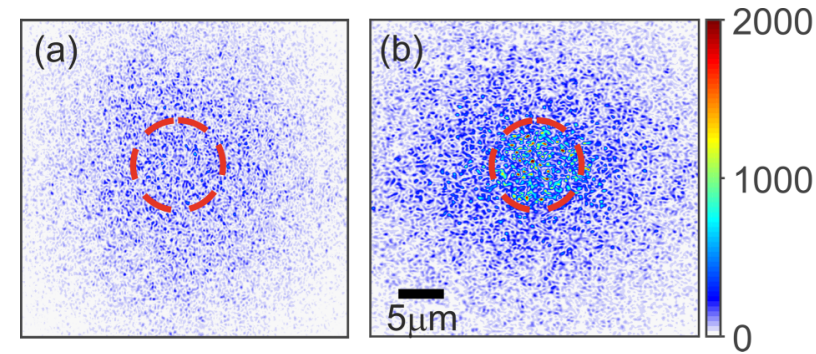

FIG. 2. Camera images of transmitted intensity at the back surface of a zinc oxide sample. In (a) and (b), unoptimized and optimized wavefronts were projected on the spatial light modulator (SLM) respectively. The optimization radius is $r_{o}=4.72 \mu \mathrm{m}$, which is indicated by the red dashed circle.

following Refs. [17,59]. $P_{\text {targ }}^{o}$ and $P_{\text {targ }}^{u}$ are the power in the target area with the optimized and unoptimized patterns, respectively. \langle\rangle denotes an ensemble-averaged power over the 100 different random phase patterns. We also quantified the enhancement $\eta_{\mathrm{tr} \text {,re }}$ of the total transmitted intensity and the total reflected intensity as

$$
\eta_{i} \equiv \frac{P_{i}^{o}}{\left\langle P_{i}^{u}\right\rangle},
$$

where $i=t r$ for transmitted light, $i=r e$ for reflected light, $P_{\mathrm{tr}}^{o}$ and $\left\langle P_{\mathrm{tr}}^{u}\right\rangle$ are the total transmitted power through the sample with the optimized and unoptimized patterns, respectively, and $P_{\mathrm{re}}^{o}$ and $\left\langle P_{\mathrm{re}}^{u}\right\rangle$ are the total reflected power through the sample with the optimized and unoptimized patterns, respectively. The enhancement of the intensity outside the optimization area is quantified as

$$
\eta_{\text {out }} \equiv \frac{P_{\mathrm{tr}}^{o}-P_{\mathrm{targ}}^{o}}{\left\langle P_{\mathrm{tr}}^{u}-P_{\mathrm{targ}}^{u}\right\rangle} .
$$

\section{RESULTS}

\section{A. Radial distribution of transmitted intensity}

In Figs. 2(a) and 2(b), we show the CCD camera images of the transmitted intensity for the unoptimized and optimized incident wavefronts, respectively. In the wavefront-shaping experiment shown in Fig. 2(b), the optimization radius is $4.72 \mu \mathrm{m}$, which corresponds to 121 pixels. The intensity in the optimization area increases significantly compared to the unoptimized intensity. The intensity outside the target area increases as well. For a better visualization of the intensity increase, we plot in Fig. 3 the radial distributions of the transmitted intensity. The radial distribution is obtained by summing the intensities within a ring with a width $\delta r$ and an inner radius of $r_{r}$ (see inset in Fig. 3). Angular averaging helps to reduce the intensity fluctuation from the speckle pattern. There is a significant intensity increase in the optimization area for both optimization radii $r_{o}=15.2 \mu \mathrm{m}$ and $r_{o}=4.7 \mu \mathrm{m}$. This intensity increase is expected since the intensity in the optimization area is the feedback to the partitioning algorithm. The peak in Fig. 3(b) is attributed to the smaller optimization radius $r_{o}=4.7 \mu \mathrm{m}$ compared to the experiment with the larger optimization radius $r_{o}=15.2 \mu \mathrm{m}$ in Fig. 3(a). With $r_{o}=15.2 \mu \mathrm{m}$, there is an enhancement over a broad area. The

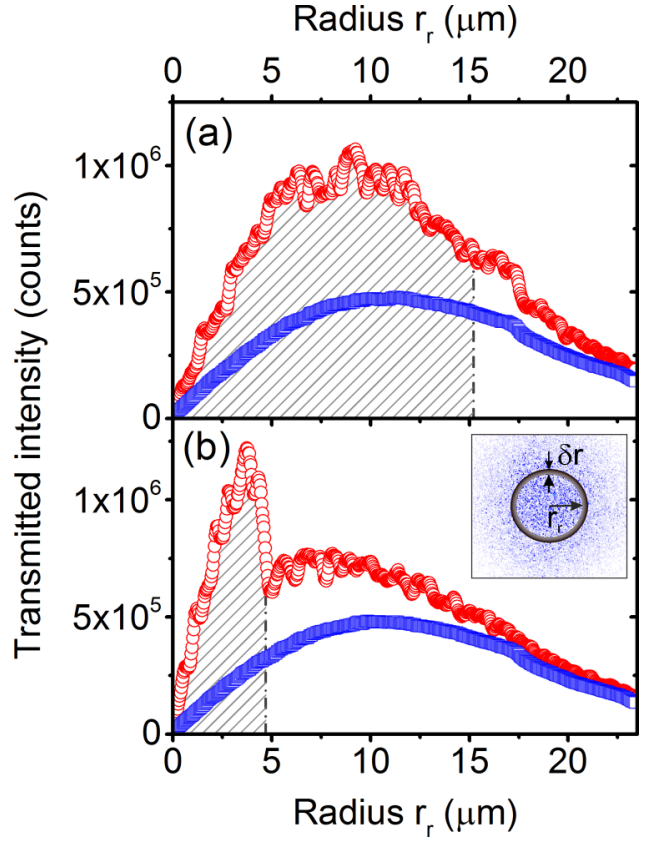

FIG. 3. Transmitted intensity vs radius $r$. The transmitted intensity is the total intensity within the circumference of a ring, which has an inner radius $r_{r}$ and ring width $\delta r$ (see inset). The optimization radii are (a) $r_{o}=15.2 \mu \mathrm{m}$ and (b) $r_{o}=4.7 \mu \mathrm{m}$. The hatched area under the curves is the optimization area. The red circles and blue squares are the intensities for the optimized and unoptimized incident wavefronts, respectively.

intensity outside the optimization area remarkably increases as well in both Figs. 3(a) and 3(b). This intensity increase agrees with the observation in Ref. [18], where the intensity outside the optimization area was observed to increase as well. We quantify the intensity enhancement inside and outside the optimization areas, and the total transmitted intensity in the next sections.

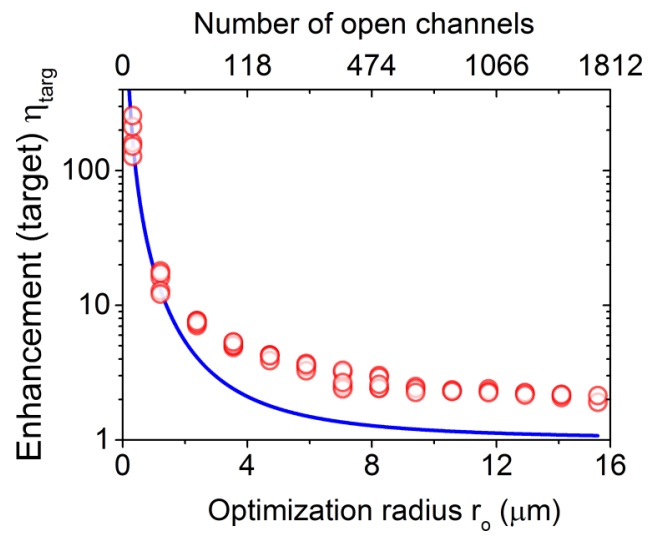

FIG. 4. Enhancement $\eta_{\text {targ }}$ in the target area vs optimization radius $r_{o}$. The red circles are the experimental data and the blue curve is an inverse square law fit following the prediction of Ref. [60]. 


\section{B. Enhancement of the intensity in the optimization area}

We plot in Fig. 4 the intensity enhancement $\eta_{\operatorname{targ}}$ in the optimization area versus the optimization radius $r_{o}$. We obtained a maximum enhancement of $257 \times$ for an optimization radius of about $0.3 \mu \mathrm{m}$, which corresponds to one speckle spot. As the optimization radius increases, $\eta_{\operatorname{targ}}$ decreases drastically down to $2 \times$ at the largest radius $r_{o}=15.2 \mu \mathrm{m}$, which corresponds to 1716 open transmission channels. In Ref. [60], the enhancement for a multiple channel optimization was found to be inversely proportional to the number of speckle spots $M$ in the optimization area. The model in [60] assumed that the optimized intensity is distributed equally to all optimized channels, which are considered statistically independent. From the model, the enhancement is expected to depend inversely on the square of the optimization radius $r_{o}$,

$$
\eta_{\operatorname{targ}}=\frac{K}{r_{o}^{2}}+1
$$

where $K$ is a constant that depends on the number of effectively controlled input channels on the sample. In Fig. 4, we show a nonlinear least squares fit to the experimental data using Eq. (5) and $K$ is the only adjustable parameter. Weighing all data points equally, the fit agrees well with the first two optimization radii $r_{o}=0.3$ and $1 \mu \mathrm{m}$, which have 1 and 7 transmission channels, respectively. Strikingly, the fit deviates from the experimental data by about a factor of 2 at large radii. This deviation signifies that there is more intensity available in the optimization channels than that predicted by Eq. (5), especially at large radii.

We discuss three possible reasons for the deviation of the theory from the experimental data. First, it is known that noise has a significant effect on the single-speckle optimization [59]. Our wavefront-shaping experiments are in the regime where shot noise is much higher than the camera and laser noises, which are about $1 \%$ and $2 \%$ respectively. According to Ref. [59], in this shot-noise regime the enhancement of a single-speckle optimization is expected to scale linearly with the total intensity in the optimization area $P_{\text {targ. Extending this }}$ model to the optimization of multiple channels, we derive

$$
\eta_{\operatorname{targ}}=\frac{K P_{\operatorname{targ}}}{r_{o}^{2}}+1=K^{\prime}+1
$$

where $K^{\prime} \equiv K C$, where $C$ is a prefactor in the relationship $P_{\text {targ }}=C r_{o}^{2}$. A constant enhancement with the radius obviously does not describe our experimental data, hence we reject this hypothesis.

Second, the observed increased enhancement might be due to intensity redistributed from the speckles outside the optimized area to speckles inside the optimized area. If this is the case, then the total transmitted intensity would be constant for all optimization radii. A third hypothesis is that there is a redistribution of intensity from the reflected speckles to the transmitted speckles. In this case, the effect of enhancing the transmitted intensity is expected to be noticeable on reflection as a reduction of the reflected intensity. We will check these latter two hypotheses in the next section.

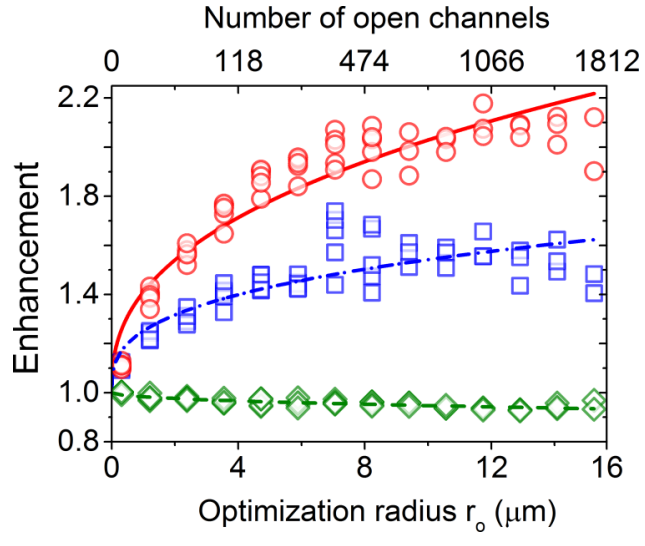

FIG. 5. Enhancement vs optimization radius $r_{o}$. The red circles are the experimental data of the enhancement of the total transmitted intensity. The blue squares are the enhancement of the intensity in the area outside the target area, and the green diamonds are the enhancement of the total reflected intensity. The curves are fits to the experimental data using Eq. (7) and the parameters are given in Table I.

\section{Change of both transmitted and reflected intensities}

The enhancements of the total transmitted intensity $\eta_{\text {tr }}$, the total reflected intensity $\eta_{\text {re }}$, and the intensity outside the optimization area $\eta_{\text {out }}$ versus the optimization radius are shown in Fig. 5 (see Appendix B for the histogram of the intensities). The enhancement of the total transmitted intensity gradually increases from about 1.1 to 2 at the largest radius of $15.2 \mu \mathrm{m}$, which is close to the $17 \mu \mathrm{m}$ large size of the detected speckle pattern. There is also an enhancement of the intensity outside the optimization area and it is about $\eta_{\text {out }} \approx 1.5$ at large radii. The enhancements of both the total transmitted intensity and the intensity of area outside the optimization nullifies the second hypothesis. In contrast to the transmitted intensity, the enhancement of the total reflected intensity slowly decreases to $\eta_{\mathrm{re}} \approx 0.93$ as the optimization radius increases. The decrease of the $\eta_{\mathrm{re}}$ is not as rapid as the increase of $\eta_{\text {tot }}$ because the reflected intensity is about nine times higher than the transmitted intensity. Therefore, a large intensity enhancement on transmission corresponds to a small intensity enhancement on reflection.

To accurately compare the decrease of $\eta_{\text {re }}$ with the increase of $\eta_{\text {tot }}$, we need to know the dependence of both terms on the optimization radius. The dependence of the enhancements on the optimization radius is unknown and we find that a power law

$$
\eta=\frac{B}{r_{o}^{n}}+1
$$

describes the experimental data well. Here, $B$ and $n$ are adjustable parameters. The fits to the experimental data are shown in Fig. 5 and the values of $B$ and $n$ obtained from the fits are shown in Table I. We obtained $n=0.4$ and $n=0.5$ for the enhancement of the total transmitted and reflected intensities, respectively, and these values are in remarkable mutual agreement.

In Fig. 6, we plot the absolute changes in the transmitted and the reflected intensities after accounting for the attenuation 
TABLE I. Values of the adjustable parameters $B$ and $n$ obtained by fitting Eq. (7) to the experimental data shown in Fig. 5 for the total transmission enhancement $\eta_{\text {tot }}$, total reflection enhancement $\eta_{\text {re }}$, and the enhancement of the intensity outside the target area $\eta_{\text {out }}$.

\begin{tabular}{lrl}
\hline \hline & \multicolumn{1}{c}{$B$} & \\
\hline$\eta_{\text {tot }}$ & 0.42 & 0.4 \\
$\eta_{\text {re }}$ & -0.02 & 0.5 \\
$\eta_{\text {out }}$ & 0.25 & 0.35 \\
\hline \hline
\end{tabular}

factor of the neutral density filters. The absolute changes in the transmitted and reflected intensities both collapse to the same curve, modeled with $n=0.45$. Therefore, the enhancement of the transmitted intensity corresponds to a decrease in the reflected intensity. This result validates the third hypothesis that there is a redistribution of intensity from the reflection speckles to the transmission speckles of the scattering medium.

\section{Radial distribution of reflected intensity}

At this point, the question arises: how does the reflected intensity change spatially? Is the change global, i.e., does the overall reflected intensity decrease uniformly, or is it local, i.e., does the intensity decrease more in the area where the transmission is enhanced? As illustrated in Fig. 7, a global change of the reflected intensity implies that the intensity redistributed to transmission is deducted equally from all the spatial channels. This is expected if all input spatial channels contribute equally to all the output spatial channels. On the other hand, a local change implies that the intensity of the spatial input channels maps one-to-one with that of the output spatial channels. The local change is expected as a result of the reflection-transmission long-range correlation predicted in Ref. [43].

To observe the type of change, we plot the radial distribution of the reflected intensity in Figs. 8(a) and 8(b) for optimization radii of 15.2 and $8.4 \mu \mathrm{m}$, respectively. In both optimization radii, the optimized (red circles) and unoptimized (blue squares) intensities match quite well from $r=0$ to about

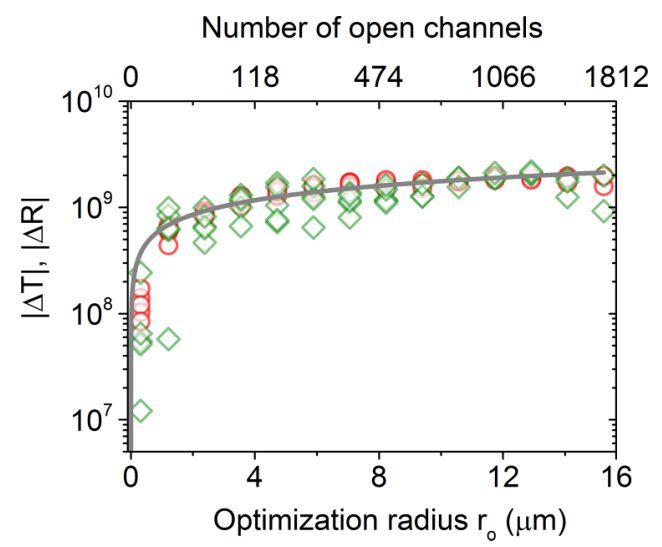

FIG. 6. Measured absolute change in the total transmitted intensity $(\Delta T)$ (red circles) and total reflected intensity $(\Delta R)$ (green diamond) versus optimization radius $r_{o}$. The solid gray curve is a fit using Eq. (7), with $B=6.3 \times 10^{8}$ and $n=0.45$.
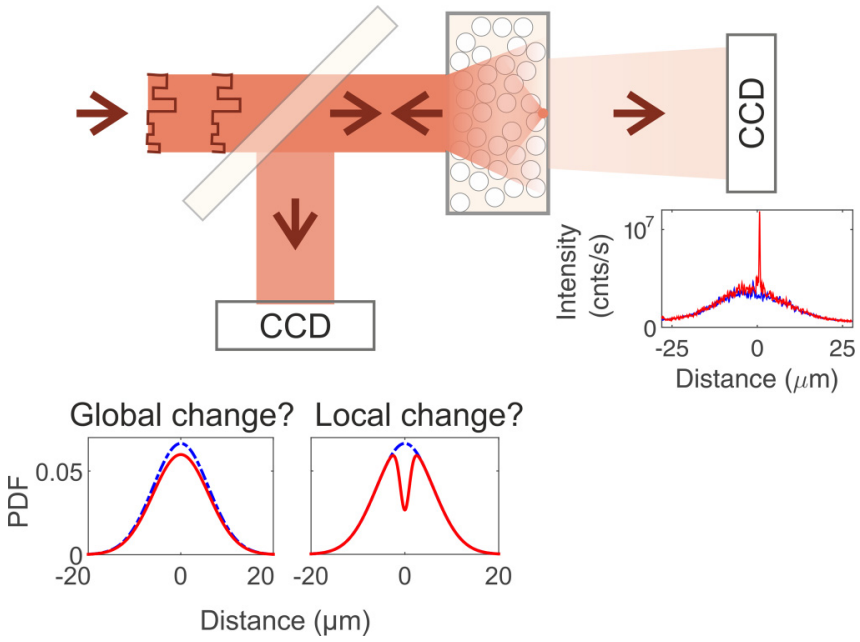

FIG. 7. Type of change that happens spatially as the reflected light decreases. The wavefront-shaped light is incident on the sample to obtain an optimized focus at the back surface of the sample. The imaged intensity data at the back surface show an enhanced peak (top inset). The question is how does the reflected intensity spatially redistribute? A global change (bottom left inset) means that there is a uniform decrease in the amplitude of the Gaussian envelope of the reflected intensity and a local change (bottom right inset) means there is a local dip in the Gaussian envelope.

$r=5 \mu \mathrm{m}$. The optimized intensity deviates asymmetrically from the unoptimized intensity between $r=5$ and $10 \mu \mathrm{m}$. At $r>10 \mu \mathrm{m}$, both intensities become equal and decrease in the same way. In order words, there is a local suppression of the optimized intensity around $r=7 \mu \mathrm{m}$.

For a proper interpretation of these results, we describe the global and local changes in the reflected intensity as follows.

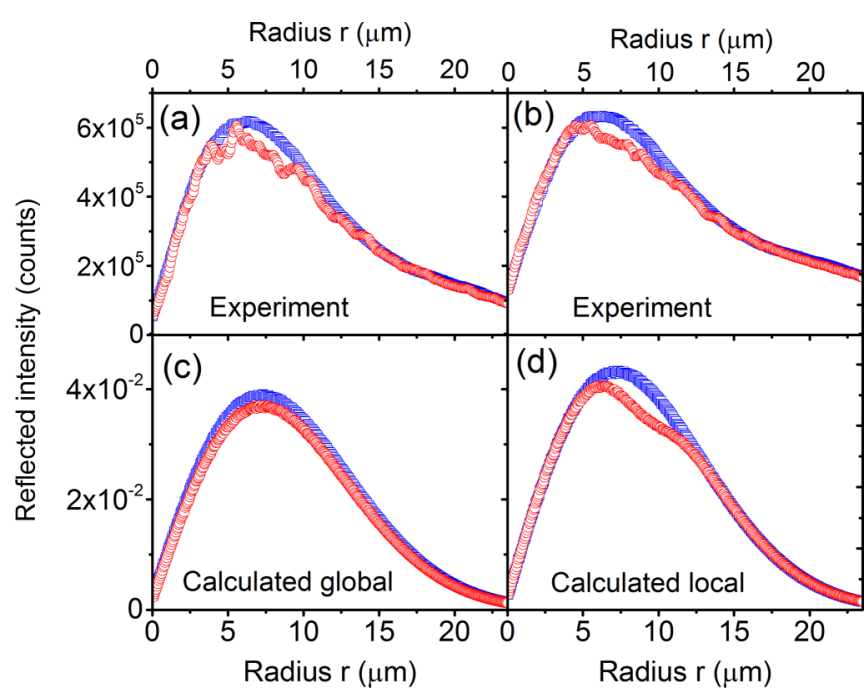

FIG. 8. Total reflected intensity integral along the circumference of a ring (see inset of Fig. 3). Blue squares are the experimental data with unoptimized wavefront projected on the SLM; red circles are the experimental data with the optimized wavefront. The optimization radii are 15.2 and $8.4 \mu \mathrm{m}$ in (a) and (b) respectively. The calculated global and local changes are plotted in (c) and (d). 
First, we model the unoptimized reflected intensity $I_{u}$ with a two-dimensional (2D) circular Gaussian function

$$
I_{u}(\boldsymbol{\rho})=\frac{A_{1}}{2 \pi \sigma_{1}^{2}} \exp \left[-\left(\frac{\left|\boldsymbol{\rho}-\boldsymbol{\rho}_{0}\right|^{2}}{2 \sigma_{1}^{2}}\right)\right] .
$$

Here, $A_{1}$ is the amplitude, $\boldsymbol{\rho}=(x, y)$ is the spatial coordinate, $\boldsymbol{\rho}_{0}=\left(x_{0}, y_{0}\right)$ is the coordinate of the center, and $\sigma_{1}$ is the standard deviation that defines the width of the function. To model both the global and local changes, we define a second 2D circular Gaussian function $I_{o}$

$$
I_{o}(\boldsymbol{\rho})=\frac{A_{2}}{2 \pi \sigma_{2}^{2}} \exp \left[-\left(\frac{\left|\boldsymbol{\rho}-\boldsymbol{\rho}_{0}-\boldsymbol{\rho}_{c}\right|^{2}}{2 \sigma_{2}^{2}}\right)\right],
$$

where $\boldsymbol{\rho}_{c}=\left(x_{c}, y_{c}\right)$ is the displacement of the center of $I_{o}$ from $x_{0}$ and $y_{0}$, respectively, and $\sigma_{2}$ is the standard deviation of $I_{o}$. We model the optimized intensity $I_{g}$ in the case of the global change as the difference between $I_{u}$ and $I_{o}$, when $\sigma_{1}=\sigma_{2}=\sigma_{g}$ and $A_{1}>A_{2}$, to get

$$
\begin{aligned}
I_{g}(\boldsymbol{\rho})= & \frac{A_{2}}{2 \pi \sigma_{g}^{2}} \exp \left[-\left(\frac{\left|\boldsymbol{\rho}-\boldsymbol{\rho}_{0}-\boldsymbol{\rho}_{c}\right|^{2}}{2 \sigma_{g}^{2}}\right)\right] \\
& -\frac{A_{1}}{2 \pi \sigma_{g}^{2}} \exp \left[-\left(\frac{\left|\boldsymbol{\rho}-\boldsymbol{\rho}_{0}\right|^{2}}{2 \sigma_{g}^{2}}\right)\right] .
\end{aligned}
$$

To model the optimized intensity $I_{l}$ in case of a local change, we use the difference between $I_{u}$ and $I_{o}$, when $\sigma_{1}>\sigma_{2}$ and $A_{1}>A_{2}$, to get

$$
\begin{aligned}
I_{l}(\boldsymbol{\rho})= & \frac{A_{2}}{2 \pi \sigma_{2}^{2}} \exp \left[-\left(\frac{\left|\boldsymbol{\rho}-\boldsymbol{\rho}_{0}-\boldsymbol{\rho}_{c}\right|^{2}}{2 \sigma_{2}^{2}}\right)\right] \\
& -\frac{A_{1}}{2 \pi \sigma_{1}^{2}} \exp \left[-\left(\frac{\left|\boldsymbol{\rho}-\boldsymbol{\rho}_{0}\right|^{2}}{2 \sigma_{1}^{2}}\right)\right] .
\end{aligned}
$$

Using Eqs. (8), (10) and (11), 2D Gaussian functions were calculated for unoptimized light and optimized light for either global or local changes, respectively. A projection of the generated functions onto the $x$ axis is shown in the bottom left and right insets of Fig. 7. Following the same procedure for the analysis of the experimental data, we obtain the radial distribution of the calculated functions by integrating the intensity along the circumference of a ring of width of $\delta r$ and inner radius of $r_{r}$.

In Figs. 8(c) and 8(d), we plot the calculated intensity versus radius for the global and local changes, respectively. The calculations were obtained using the parameters listed in Table II. For the case of a global change in Fig. 8(c), the optimized reflected intensity has roughly the same shape as the unoptimized one. In the calculated curve for the global change, there is no local suppression of the reflected intensity around $r=7 \mu \mathrm{m}$, leading to a qualitative mismatch with the experimental data. This holds true for any realistic value of the adjustable parameters. The calculated curve for a local change, shown in Fig. 8(d), does exhibit a characteristic dip around $r=7 \mu \mathrm{m}$, very similar to the one in the experimental data. Hence, the experimental data clearly support the model that optimization of the transmission results in a local change in the reflection.
TABLE II. Parameters used in Eqs. (10) and (11).

\begin{tabular}{lcc}
\hline \hline & Parameters & Values \\
\hline \multirow{4}{*}{ Fixed } & $A_{1}$ & 1 \\
& $A_{2}$ & 0.05 \\
& $\sigma_{1}$ & $7.35 \mu \mathrm{m}$ \\
& $\left(x_{0}, y_{0}\right)$ & $(45.5,45.5) \mu \mathrm{m}$ \\
\hline Adjusted & $\left(x_{c}, y_{c}\right)$ & $(6.4,6.4) \mu \mathrm{m}$ \\
& $\sigma_{2}$ & $2.1 \mu \mathrm{m}$ \\
\hline \hline
\end{tabular}

In Table II, the adjusted parameters were chosen to reproduce the qualitative features of the experimental data. We note that our analytical model is intended to highlight qualitative features related to correlations between reflected and transmitted intensities. The Gaussian approximation to the spot shape is not suitable for quantitative parameter estimation. One important parameter is the displacement $\left(x_{c}, y_{c}\right)$ between the optimization area and the center of the diffuse unoptimized spot. The shape of the experimental data is best reproduced assuming a displacement $\left(x_{c}, y_{c}\right)=(6.4,6.4) \mu \mathrm{m}$, which is likely due to thermal drift in the apparatus, since the experiment took several days.

\section{SUMMARY}

We have experimentally shown that as the optimization radius increases, the enhancement of the total transmitted intensity increases, while simultaneously the total reflected intensity decreases. We also find that the enhancement of the intensity outside the optimization area increases as the optimization radius increases. From the radial intensity distribution of the reflected intensity, we find evidence that there is a local decrease in the reflected intensity rather than a global decrease. The local decrease confirms that the transmitted and reflected intensities are spatially correlated as recently predicted by Fayard et al. [43]. Our results have prospects in extending the applications of wavefront shaping to increase the total transmitted intensity through the rough layer on top of the silicon absorber in a solar cell. Our results are also interesting for multichannel quantum secure communication $[36,48,49]$, where enhanced intensities are desired in multiple transmission channels; for transmitting arbitrary intensity distribution through a scattering medium [52]; and imaging through an opaque medium [34,50,51].

\section{ACKNOWLEDGMENTS}

We thank Duygu Akbulut, Ad Lagendijk, Ivo Vellekoop, Ravitej Uppu, and Tom Wolterink for discussions, and Cock Harteveld for technical assistance. This project is part of the research program of the Stichting voor Fundamenteel Onderzoek der Materie (FOM) FOM-program Stirring of Light!, which is part of the Nederlandse Organisatie voor Wetenschappelijk Onderzoe (NWO). We acknowledge support from NWO-Vici, DARPA, ERC, and STW. 


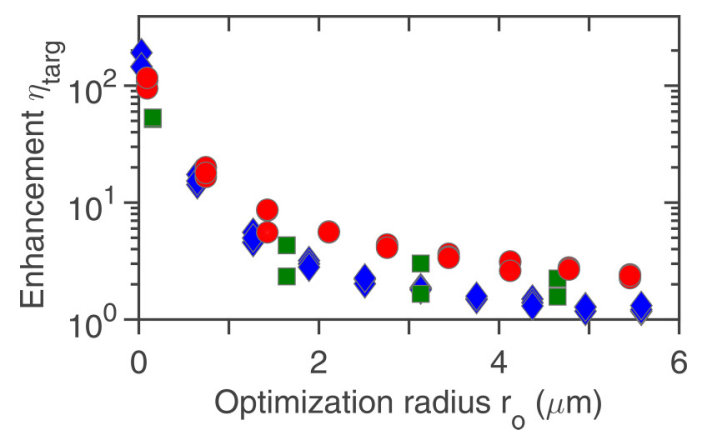

FIG. 9. Enhancement $\eta_{\text {targ }}$ in optimization target vs the optimization radius $r_{o}$ for different algorithms. The red circles, green squares, and blue diamonds are the data points for the partitioning, genetic, and stepwise sequential algorithms, respectively. The partitioning algorithm outperforms the other algorithms.

\section{APPENDIX A: COMPARING WAVEFRONT-SHAPING ALGORITHMS FOR LARGE-AREA OPTIMIZATION}

For the optimization of intensity in large areas, we investigated three different wavefront-shaping algorithms: the stepwise sequential, partitioning, and genetic algorithms. The details on how these algorithms work are described in Refs. [54,61]. First, the sequential algorithm modulates the phase of the segments of the SLM one by one and combines them at the end of optimization. Second, the partitioning algorithm modulates the phase of $50 \%$ of the segments simultaneously and keeps the optimized phases on the SLM. The modulated segments are chosen randomly at each step. A better performance of the partitioning algorithm is expected because a larger number of segments is controlled simultaneously, and this is expected to yield a significant change in the target signal compared to the sequential algorithm. Third, the genetic algorithm begins by creating a population of random phase masks, which are ranked using the measured enhancement. The phase masks are combined using a weight proportional to the enhancement and then further mutated to create new phase masks. The new phase masks are measured and replace the low ranking members of the population. As the whole steps are repeated, the average enhancement of the population increases and finally converges.

We have performed wavefront-shaping experiments to compare the enhancement of the three algorithms. In Fig. 9, we plot
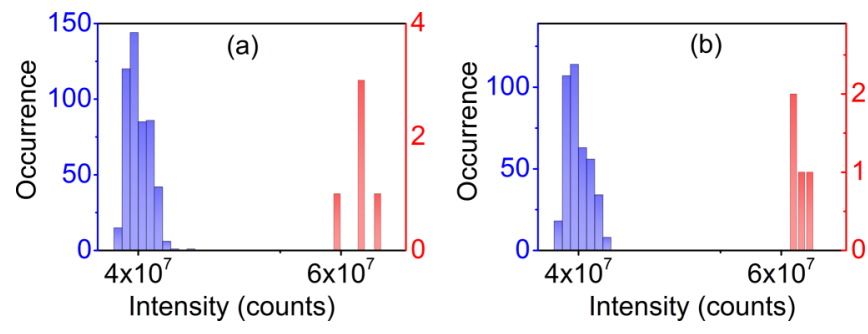

FIG. 10. Histogram of the transmitted intensities of unoptimized light (blue) and optimized light (red) for different optimization radii: (a) $r_{o}=7 \mu \mathrm{m}$ and (b) $r_{o}=8 \mu \mathrm{m}$.

the enhancement $\eta_{\text {targ }}$ in the optimization area versus the optimization radius $r_{o}$. The enhancement decreases with increasing radius for all algorithms, as expected. With the partitioning algorithm, the enhancement is on average approximately $85 \%$ higher than for the sequential algorithm. The partitioning algorithm outperforms the sequential algorithm because of the larger modulation signal in the optimization area. Furthermore, the partitioning algorithm has an enhancement that is $80 \%$ higher than for the genetic algorithm. We expected a similar performance of the genetic algorithm and the partitioning algorithm, since a comparable number of segments are simultaneously controlled in the two algorithms. We attribute the lower performance to the fact that the genetic algorithm requires a large number of experimental parameters, which might differ for different optimization radii. A further detailed study of using genetic algorithm for large-area optimization is needed. We have therefore chosen to use the partitioning algorithm since it shows a better performance than the other two algorithms.

\section{APPENDIX B: HISTOGRAM OF OPTIMIZED AND UNOPTIMIZED LIGHT}

In the experiments, we average over 100 realizations of unoptimized intensity and over two to five realizations of optimized intensity. In Fig. 10, we show the histogram of the transmitted intensities for optimized and unoptimized light in the experiments with two different radii. There are five and four wavefront-shaping experiments in Figs. 10(a) and 10(b) respectively. There is clearly no overlap between the distribution of the unoptimized and optimized intensities. This observation shows that the intensity is truly enhanced by wavefront shaping.
[1] J. W. Goodman, Speckle Phenomena in Optics: Theory and Applications (Roberts, Englewood, CO, 2007).

[2] B. Shapiro, Large Intensity Fluctuations for Wave Propagation in Random Media, Phys. Rev. Lett. 57, 2168 (1986).

[3] M. J. Stephen and G. Cwilich, Intensity Correlation Functions and Fluctuations in Light Scattered from a Random Medium, Phys. Rev. Lett. 59, 285 (1987).

[4] S. Feng, C. Kane, P. A. Lee, and A. D. Stone, Correlations and Fluctuations of Coherent wave Transmission through Disordered Media, Phys. Rev. Lett. 61, 834 (1988).

[5] J. X. Zhu, D. J. Pine, and D. A. Weitz, Internal reflection of diffusive light in random media, Phys. Rev. A 44, 3948 (1991).
[6] R. Berkovits and S. Feng, Correlations in coherent multiple scattering, Phys. Rep. 238, 135 (1994).

[7] F. Scheffold and G. Maret, Universal Conductance Fluctuations of Light, Phys. Rev. Lett. 81, 5800 (1998).

[8] M. C. W. van Rossum and Th. M. Niewenhuizen, Multiple scattering of classical waves, Rev. Mod. Phys. 71, 313 (1999).

[9] B. Shapiro, New Type of Intensity Correlation in Random Media, Phys. Rev. Lett. 83, 4733 (1999).

[10] M. Birowosuto, S. Skipetrov, W. L. Vos, and A. P. Mosk, Observation of Spatial Fluctuations of the Local Density of States in Random Media, Phys. Rev. Lett. 105, 013904 (2010). 
[11] M. P. van Albada and A. Lagendijk, Observation of Weak Localization of Light in a Random Medium, Phys. Rev. Lett. 55, 2692 (1985).

[12] P.-E. Wolf and G. Maret, Weak Localization and Coherent Backscattering of Photons in Disordered Media, Phys. Rev. Lett. 55, 2696 (1985).

[13] C. A. Müller, T. Jonckheere, C. Miniatura, and D. Delande, Weak localization of light by cold atoms: The impact of quantum internal structure, Phys. Rev. A 64, 053804 (2001).

[14] P. W. Anderson, Absence of diffusion in certain random lattices, Phys. Rev. 109, 1492 (1958).

[15] C. Conti, L. Angelani, and G. Ruocco, Light diffusion and localization in three-dimensional nonlinear disordered media, Phys. Rev. A 75, 033812 (2007).

[16] I. Freund, Looking through walls and around corners, Physica A 168, 49 (1990).

[17] I. M. Vellekoop and A. P. Mosk, Focusing coherent light through opaque strongly scattering media, Opt. Lett. 32, 2309 (2007).

[18] I. M. Vellekoop and A. P. Mosk, Universal Optimal Transmission of Light through Disordered Materials, Phys. Rev. Lett. 101, 120601 (2008).

[19] S. M. Popoff, A. Goetschy, S. F. Liew, A. D. Stone, and H. Cao, Coherent Control of Total Transmission of Light through Disordered Media, Phys. Rev. Lett. 112, 133903 (2014).

[20] M. Davy, Z. Shi, and A. Z. Genack, Focusing through random media: Eigenchannel participation number and intensity correlation, Phys. Rev. B 85, 035105 (2012).

[21] A. P. Mosk, A. Lagendijk, G. Lerosey, and M. Fink, Controlling waves in space and time for imaging and focusing in complex media, Nat. Photon. 6, 283 (2012).

[22] I. M. Vellekoop, Feedback-based wavefront shaping, Opt. Express 23, 12189 (2015).

[23] A. Derode, P. Roux, and M. Fink, Robust Acoustic Time Reversal with High-Order Multiple Scattering, Phys. Rev. Lett. 75, 4206 (1995).

[24] G. Lerosey, J. de Rosny, A. Tourin, A. Derode, G. Montaldo, and M. Fink, Time Reversal of Electromagnetic Waves, Phys. Rev. Lett. 92, 193904 (2004).

[25] G. Lerosey, J. de Rosny, A. Tourin, and M. Fink, Focusing beyond the diffraction limit with far-field time reversal, Science 315, 1120 (2007).

[26] E. N. Leith and J. Upatnieks, Holographic imagery through diffusing media, J. Opt. Soc. Am. 56, 523 (1966).

[27] D. R. Dowling and D. R. Jackson, Narrow-band performance of phase-conjugate arrays in dynamic random media, J. Acoust. Soc. Am. 91, 3257 (1992).

[28] Z. Yaqoob, D. Psaltis, M. S. Feld, and C. Yang, Optical phase conjugation for turbidity suppression in biological samples, Nat. Photonics 2, 110 (2008).

[29] M. Kim, Y. Choi, C. Yoon, W. Choi, J. Kim, Q.-H. Park, and W. Choi, Maximal energy transport through disordered media with the implementation of transmission eigenchannels, Nat. Photonics 6, 581 (2012).

[30] S. M. Popoff, G. Lerosey, R. Carminati, M. Fink, A. C. Boccara, and S. Gigan, Measuring the Transmission Matrix in Optics: An Approach to the Study and Control of Light Propagation in Disordered Media, Phys. Rev. Lett. 104, 100601 (2010).

[31] S. M. Popoff, G. Lerosey, M. Fink, A. C. Boccara, and S. Gigan, Controlling light through optical disordered media: Transmission matrix approach, New J. Phys. 13, 123021 (2011).
[32] Y. M. Wang, B. Judkewitz, C. A. DiMarzio, and C. Yang, Deeptissue focal fluorescence imaging with digitally time-reversed ultrasound-encoded light, Nat. Commun. 3, 928 (2012).

[33] K. Si, R. Fiolka, and M. Cui, Fluorescence imaging beyond the ballistic regime by ultrasound-pulse-guided digital phase conjugation, Nat. Photon. 6, 657 (2012).

[34] J. Bertolotti, E. G. van Putten, C. Blum, A. Lagendijk, W. L. Vos, and A. P. Mosk, Non-invasive imaging through opaque scattering layers, Nature 491, 232 (2012).

[35] T. Čižmár, M. Mazilu, and K. Dholakia, In situ wavefront correction and its application to micromanipulation, Nat. Photon. 4, 388 (2010).

[36] S. R. Huisman, T. Huisman, T. A. W. Wolterink, A. P. Mosk, and P. W. H. Pinkse, Programmable multiport optical circuits in opaque scattering materials, Opt. Express 23, 3102 (2015).

[37] J.-H. Park, C. Park, H. Yu, Y.-H. Cho, and Y. Park, Dynamic active wave plate using random nanoparticles, Opt. Express 20, 17010 (2012).

[38] Y. Guan, O. Katz, E. Small, J. Zhou, and Y. Silberberg, Polarization control of multiply scattered light through random media by wavefront shaping, Opt. Lett. 37, 4663 (2012).

[39] J.-H. Park, C. Park, H. Yu, Y.-H. Cho, and Y. Park, Active spectral filtering through turbid media, Opt. Lett. 37, 3261 (2012).

[40] E. Small, O. Katz, Y. Guan, and Y. Silberberg, Spectral control of broadband light through random media by wavefront shaping, Opt. Lett. 37, 3429 (2012).

[41] R. Horstmeyer, B. Judkewitz, I. M. Vellekoop, S. Assawaworrarit, and C. Yang, Physical key-protected one-time pad, Sci. Rep. 3, 3543 (2013).

[42] S. A. Goorden, M. Horstmann, A. P. Mosk, B. Škoríc, and P. W. H. Pinkse, Quantum-secure authentication of a physical unclonable key, Optica 1, 421 (2014).

[43] N. Fayard, A. Cazé, R. Pierrat, and R. Carminati, Intensity correlations between reflected and transmitted speckle patterns, Phys. Rev. A 92, 033827 (2015).

[44] W. Choi, M. Kim, D. Kim, C. Yoon, C. Fang-Yen, Q.-H. Park, and W. Choi, Preferential coupling of an incident wave to reflection eigenchannels of disordered media, Sci. Rep. 5, 11393 (2015).

[45] J. A. Levitt and W. H. Weber, Materials for luminescent greenhouse solar collectors, Appl. Opt. 16, 2684 (1977).

[46] A. Polman and H. Atwater, Photonic design principles for ultrahigh-efficiency photovoltaics, Nat. Mater. 11, 174 (2012).

[47] F. T. Si, D. Y. Kim, R. Santbergen, H. Tan, R. A. C. M. M. van Swaaij, A. H. M. Smets, O. Isabella, and M. Zeman, Quadruplejunction thin-film silicon-based solar cells with high open-circuit voltage, Appl. Phys. Lett. 105, 063902 (2014).

[48] H. Defienne, M. Barbieri, I. A. Walmsley, B. J. Smith, and S. Gigan, Two-photon quantum walk in a multimode fiber, Sci. Adv. 2, e1501054 (2016).

[49] T. A. W. Wolterink, R. Uppu, G. Ctistis, W. L. Vos, K.-J. Boller, and P. W. H. Pinkse, Programmable two-photon quantum interference in $10^{3}$ channels in opaque scattering media, Phys. Rev. A 93, 053817 (2016).

[50] I. M. Vellekoop and C. M. Aegerter, Scattered light fluorescence microscopy: Imaging through turbid layers, Opt. Lett. 35, 1245 (2010).

[51] O. Katz, P. Heidmann, M. Fink, and S. Gigan, Noninvasive single-shot imaging through scattering layers and 
around corners via speckle correlations, Nat. Photon 8, 784 (2014).

[52] F. Dickey, Laser Beam Shaping: Theory and Techniques, 2nd ed. (CRC, Boca Raton, FL, 2014).

[53] E. van Putten, Disorder-enhanced imaging with spatially controlled light, Ph.D. thesis, University of Twente, 2011, available at www.complexphotonicsystems.com.

[54] I. M. Vellekoop and A. P. Mosk, Phase control algorithms for focusing light through turbid media, Opt. Commun. 281, 3071 (2008).

[55] B. R. Anderson, R. Gunawidjaja, and H. Eilers, Effect of experimental parameters on optimal transmission of light through opaque media, Phys. Rev. A 90, 053826 (2014).

[56] O. S. Ojambati, H. Yilmaz, A. Lagendijk, A. P. Mosk, and W. L. Vos, Coupling of energy into the fundamental diffusion mode of a complex nanophotonic medium, New J. Phys. 18, 043032 (2016).
[57] J. F. de Boer, Optical fluctuations on the transmission and reflection of mesoscopic systems, Ph.D. thesis, University of Amsterdam, 1995, available at www.complexphotonicsystems.com.

[58] D. Akbulut, Measurements of strong correlations in the transport of light through strongly scattering materials, Ph.D. thesis, University of Twente, 2013, available at www.complexphotonicsystems.com.

[59] H. Yilmaz, W. L. Vos, and A. P. Mosk, Optimal control of light propagation through multiple-scattering media in the presence of noise, Biomed. Opt. Express 4, 1759 (2013).

[60] I. M. Vellekoop, E. G. van Putten, A. Lagendijk, and A. P. Mosk, Demixing light paths inside disordered metamaterials, Opt. Express 16, 67 (2008).

[61] D. B. Conkey, A. N. Brown, A. M. Caravaca-Aguirre, and R. Piestun, Genetic algorithm optimization for focusing through turbid media in noisy environments, Opt. Express 20, 4840 (2012). 